\title{
New Structure Design of Magnet for 1 MJ HTS SMES
}

\author{
Wei Wei ${ }^{1}$, Yinghong Luo ${ }^{1}$, Liying Han $^{2}$ \\ ${ }^{1}$ College of Automation and Electrical Engineering, Lanzhou jiaotong University, \\ Lanzhou, China \\ ${ }^{2}$ Marx Institute, Hubei University of Technology, Wuhan, China \\ darkview@126.com,lyhdd-321@163.com
}

\begin{abstract}
In recent years, the superconducting technology is popular in our production and life. An increasing number of researchers and institutions participate in study of the superconducting magnetic energy storage (SMES) system. SMES is widely used in power supply system, new energy system and pulsed power supply system. However, the leakage magnetic field of traditional SMES is more serious, which limited the application in engineering. This paper proposes an improved scheme for the $1 \mathrm{MJ}$ single-solenoid structure magnet by using the actively shielding theory. Moreover, by using particle swarm optimization, the structural parameters of the superconducting magnets are optimized respectively. Finally, the ANSYS modeling simulation is built by these structural parameters, all simulation data are analyzed and compared.
\end{abstract}

Keywords: SMES, HTS, Actively shielding, Multi solenoid type magnet, ANSYS

\section{Introduction}

Superconducting Magnetic Energy Storage (SMES) System is one of power system devices. It can store energy by the superconducting magnetic and can release energy to the power system. The application prospect of SMES is very extensive. SMES can enhance the stability of power system when it is used in power supply system. It can also improve the power output characteristics when it is used in decentralized power systems; Furthermore, it can be used as the energy storage component in some pulsed-power devices[1-3].

With an increasing number of SMES, its electromagnetic radiation problem becomes more and more serious. The leakage magnetic field of traditional SMES is more serious, limiting the application in engineering. So it is necessary to research the shielding of leakage magnetic fields in SMES. We take the 1 MJ SMES for example. In order to reduce the leakage magnetic field, the structure of single solenoid type superconducting magnet is analyzed. At the same time, this paper designs a new kind of leakage magnetic shielding magnets based on the principle of the actively shielding theory of SMES.

\section{Selection of HTS Wires}

High-temperature superconductors (HTS) are materials that behave as superconductors at unusually high temperatures. The development of HTS has gone through three stages. At the first stages, the bismuth strontium calcium copper oxide (BSCCO) has been used in SMES[4]. At the second stages, the yttrium barium copper oxide (YBCO) has been used in SMES. The 
$\mathrm{MgB} 2$ is the newest HTS and it has been used at the third stages. Currently, the BSCCO wires are easy to be produced and they are huge applications for many SMES. So, the BSCCO wires from the ASC company of the USA are selected to produce SMES in this paper. The cross-sectional area of single BSCCO wire is $0.3 \times 4.1 \mathrm{~mm}^{2}$.

The critical current of BSCCO wire depends on the distribution of the flux density in the SMES magnet. In order to maintain SMES working properly, the critical current of SMES magnet should befit the working current of SMES magnet. According to this, ten BSCCO wires are paralleled as the materials of SMES magnet. The cross-sectional area of the multi BSCCO wires is $4.4 \times 5.4 \mathrm{~mm}^{2}$. We set the working current of SMES magnet as $1000 \mathrm{~A}$ and the current of each single wire is $100 \mathrm{~A}$ [9-10]. The working current of SMES magnet is proved to be reasonable by simulation data.

\section{Actively Shielding Theory of SMES}

Currently, there are two different types in shielding method of SMES magnets. One is passively shielding method; The other is actively shielding method. The passively shielding method is a leakage magnetic shielding method that leakage flux density in SMES magnet is reduced by setting ferromagnetic materials next to SMES[2-5]. Based on the principle actively shielding theory, the actively shielding method can help us reduce the leakage flux density in SMES magnet. Although the passively shielding method is easier than the actively shielding method, a lot of ferromagnetic materials may be wasted by the passively shielding method. With the development of SMES, actively shielding method get more and more attention.

Since the leakage magnetic field of a single solenoid type SMES with high energy cannot effectively decrease, the combination of several magnets allows us to reduce the leakage magnetic field. When the magnetic dipole moment of magnets approaches to zero, the leakage magnetic field of SMES can rapidly decrease[7-9].

The magnetic dipole moment of a single solenoid $\mathrm{m}$ can be expressed as follow

$$
m=\omega S I
$$

where $\omega=$ the turns of solenoid; $S=$ the area enclosed by magnet; and $I=$ current of the solenoid. In addition, $m$ depends on the direction of current. When several solenoid are combined, $m=$ the sum of magnetic dipole moment of each solenoid.

\section{Design of Magnet for SMES}

Since the BSCCO wires are expensive, the least materials is the target we owe to. We can measure the cost of materials by calculating the volume of SMES magnets.

The magnetic energy stored by the SMES magnet $E$ can be expressed as follow

$$
E=\frac{1}{2} L I^{2}
$$

where $L=$ inductance of SMES magnet; and $I=$ current of the magnet. Since we set current of SMES magnet as $1000 \mathrm{~A}$ and the magnetic energy stored by the SMES magnet is 1 MJ, the inductance of SMES magnets in this paper should be set as $2 \mathrm{H}$. 


\subsection{Inductance of Several Magnets}

4.1.1. Third-order headings: The model of single solenoid type magnet is shown as Figure 1, where $d=$ the average diameter of solenoid; $r=$ the thickness of solenoid; $a=$ the height of solenoid; $R_{1}=$ the inside radius of solenoid; and $R_{2}=$ the outer radius.

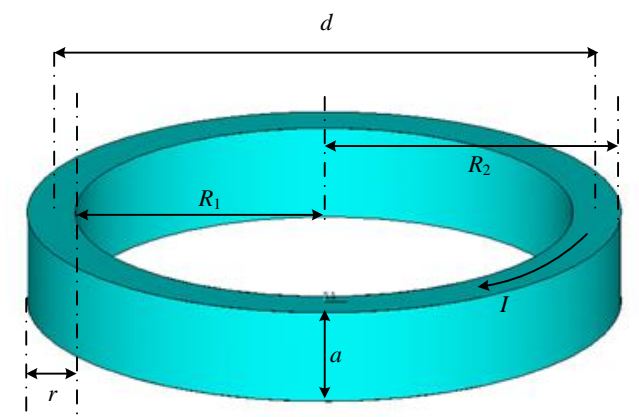

Figure 1. Model of Single Solenoid Type Magnet

The inductance of the single solenoid type magnet $L_{\mathrm{s}}$ can be expressed as follow[6]

$$
\begin{aligned}
& L_{\mathrm{s}}=\frac{\mu_{0}}{4 \pi} \omega^{2} d \cdot 2 \pi\left\{\left[1+\frac{1}{8}\left(\frac{a}{d}\right)^{2}-\frac{1}{64}\left(\frac{a}{d}\right)^{4}\right] \ln \frac{4 d}{a}-\frac{1}{2}+\frac{1}{32}\left(\frac{a}{d}\right)^{2}+\frac{1}{96}\left(\frac{a}{d}\right)^{4}\right\} \\
& -\frac{\mu_{0}}{2} \omega^{2} d\left\{\left[\frac{\pi}{3} \cdot \frac{r}{a}-\frac{25}{72} \cdot\left(\frac{r}{a}\right)^{2}-\frac{1}{8} \cdot\left(\frac{r}{d}\right)^{2}+\frac{19}{768}\left(\frac{a}{d}\right)^{2} \cdot\left(\frac{r}{d}\right)^{2}-\frac{1}{180} \cdot\left(\frac{r}{a}\right)^{4}+\right.\right. \\
& \left.\left.\frac{67}{7200}\left(\frac{r}{a}\right)^{2} \cdot\left(\frac{r}{d}\right)^{2}-\frac{17}{3840}\left(\frac{r}{d}\right)^{4}\right] \cdot \ln \frac{4 d}{a}-\left[\frac{1}{6} \cdot\left(\frac{r}{a}\right)^{2}-\frac{1}{120}\left(\frac{r}{a}\right)^{2}\left(\frac{r}{d}\right)^{2}\right] \ln \frac{a}{r}\right\}
\end{aligned}
$$

where $\omega=$ the turns of solenoid; $\mu_{0}=4 \pi \times 10^{-7} \mathrm{H} / \mathrm{m}$.

4.1.2. Axially Displaced Solenoid Arrangement: The model of axially displaced solenoid arrangement is shown as Figure 2, where each solenoid has the same size. We call them NO. 1 and NO. 3 solenoid.

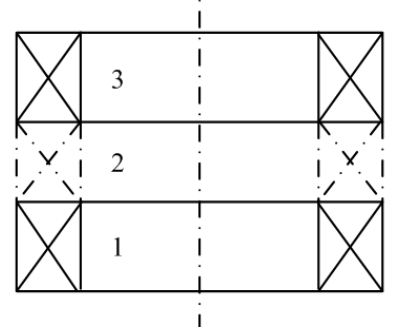

Figure 2. Model of Axially Displaced Solenoid Arrangement

We assume that there is a solenoid called NO. 2 between NO. 1 and NO. 3 solenoid. NO. 2 solenoid is shown in Figure 2. The mutual inductance between NO. 1 and NO. 3 solenoid can be expressed as follow[6]

$$
M_{13}=\frac{1}{2}\left(L_{123}+L_{2}-L_{12}-L_{23}\right)
$$


where $L_{123}=$ self-inductance of the magnet combined by NO. 1, NO. 2, NO. 3 solenoid; $L_{2}$ $=$ self-inductance of NO. 2 solenoid; $L_{12}=$ self-inductance of the magnet combined by NO. 1 and NO. 2 solenoid; $L_{23}=$ self-inductance of the magnet combined by NO. 2 and NO. 3 solenoid.

4.1.3. Radially displaced solenoid arrangement: The model of radially displaced solenoid arrangement is shown as Figure 3, where each solenoid has the same size.

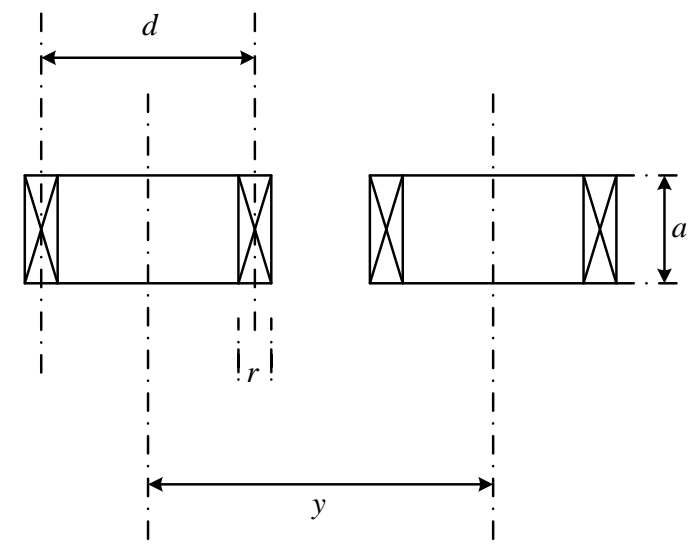

Figure 3. Model of Radially Displaced Solenoid Arrangement

As shown in the figure, the mutual inductance between two solenoid can be expressed as follow[6]

$$
M=\frac{\pi}{32} \mu_{0} \omega^{2} \frac{d^{4}}{a^{2}}\left(\frac{Z_{1}}{b_{1}}-\frac{Z_{2}}{b_{2}}\right)
$$

where $\omega=$ the turns of single solenoid; $\mu_{0}=4 \pi \times 10^{-7} \mathrm{H} / \mathrm{m} ; Z_{1}, Z_{2}, b_{1}$ and $b_{2}$ can be expressed as follow[6]

$$
\begin{aligned}
& Z_{k}=\rho_{2}^{2}-\frac{1}{2} \alpha_{k}^{2} \rho_{2} \rho_{4} P_{2}\left(\gamma_{k}\right)+\frac{1}{8} \alpha_{k}^{4}\left(2 \rho_{6} \rho_{2}+3 \rho_{4}^{2}\right) P_{4}\left(\gamma_{k}\right)-\frac{5}{35} \alpha_{k}^{6}\left(\rho_{8} \rho_{2}+\right. \\
& \left.6 \rho_{6} \rho_{4}\right) P_{6}\left(\gamma_{k}\right)+\frac{7}{64} \alpha_{k}^{8}\left(\rho_{10} \rho_{2}+10 \rho_{8} \rho_{4}+10 \rho_{6}^{2}\right) P_{8}\left(\gamma_{k}\right)+\cdots \cdots \\
& \left\{\begin{array}{l}
b_{1}=\sqrt{a^{2}+y^{2}} \\
b_{2}=y
\end{array}\right.
\end{aligned}
$$

The unknowns in equation (6) can be expressed as follow[6] 


$$
\left\{\begin{array}{l}
\rho_{n}=1+\frac{n(n-1)}{3 !} \rho^{2}+\frac{n(n-1)(n-2)(n-3)}{5 !} \rho^{4} \\
+\cdots+\frac{n(n-1)(n-2) \cdots \cdot 2 \cdot 1}{(n+1) !} \rho^{n} \\
\rho=\frac{r}{d} \\
\alpha_{1}=\frac{d}{2 b_{1}} \\
\alpha_{2}=\frac{d}{2 y} \\
\gamma_{1}=\frac{a}{b_{1}} \\
\gamma_{2}=0 \\
P_{n}(x)=\frac{1}{2^{n}(n !)} \cdot \frac{\mathrm{d}^{n}}{\mathrm{~d} x^{n}}\left(x^{2}-1\right)^{n}
\end{array}\right.
$$

4.1.4. Complexly Displaced Solenoid Arrangement: The model of complexly displaced solenoid arrangement is shown as Figure 4, where each solenoid has the same size.

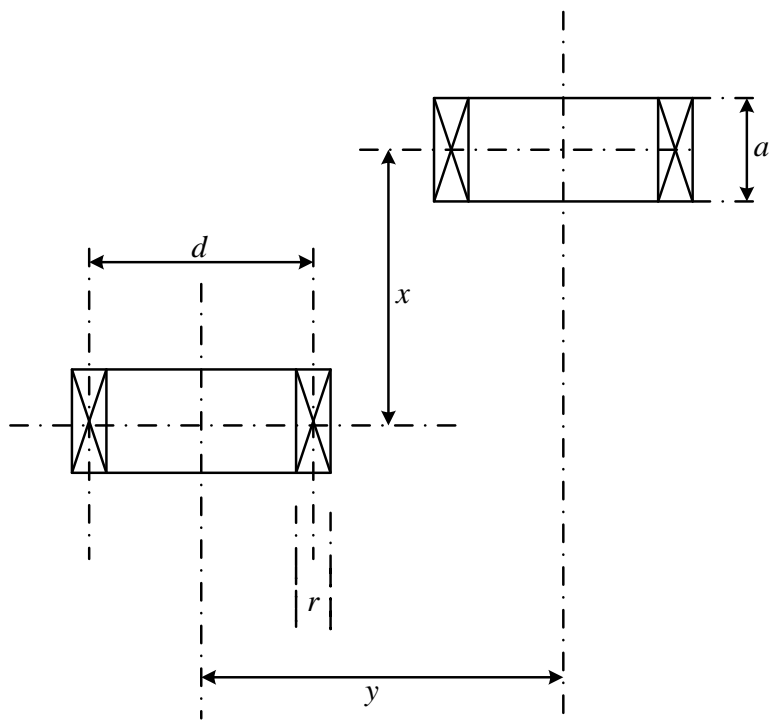

Figure 4. Model of Complexly Displaced Solenoid Arrangement

As shown in the figure, the mutual inductance between two solenoid can be expressed as follow[6]

$$
M=\frac{\pi}{64} \mu_{0} \omega^{2} \frac{d^{4}}{a^{2}}\left(\frac{Z_{1}}{b_{1}}-\frac{Z_{2}}{b_{2}}-\frac{Z_{3}}{b_{3}}+\frac{Z_{4}}{b_{4}}\right)
$$

where $\omega=$ the turns of single solenoid; $\mu_{0}=4 \pi \times 10^{-7} \mathrm{H} / \mathrm{m} ; Z_{1}, Z_{2}, Z_{3}, Z_{4}, b_{1}, b_{2}, b_{3}$ and $b_{4}$ can be expressed as follow[6] 


$$
\begin{aligned}
& Z_{k}=\rho_{2}^{2}-\frac{1}{2} \alpha_{k}^{2} \rho_{2} \rho_{4} P_{2}\left(\gamma_{k}\right)+\frac{1}{8} \alpha_{k}^{4}\left(2 \rho_{6} \rho_{2}+3 \rho_{4}^{2}\right) P_{4}\left(\gamma_{k}\right)-\frac{5}{35} \alpha_{k}^{6}\left(\rho_{8} \rho_{2}\right. \\
& \left.+6 \rho_{6} \rho_{4}\right) P_{6}\left(\gamma_{k}\right)+\frac{7}{64} \alpha_{k}^{8}\left(\rho_{10} \rho_{2}+10 \rho_{8} \rho_{4}+10 \rho_{6}^{2}\right) P_{8}\left(\gamma_{k}\right)+\cdots \cdots \\
& b_{k}=\sqrt{c_{k}^{2}+y^{2}}
\end{aligned}
$$

The unknowns in equation (10) and equation (11) can be expressed as follow[6]

$$
\left\{\begin{array}{l}
\rho_{n}=1+\frac{n(n-1)}{3 !} \rho^{2}+\frac{n(n-1)(n-2)(n-3)}{5 !} \rho^{4} \\
+\cdots+\frac{n(n-1)(n-2) \cdots \cdot 2 \cdot 1}{(n+1) !} \rho^{n} \\
\rho=\frac{r}{d} \\
\alpha_{k}=\frac{d}{2 b_{k}} \\
\gamma_{k}=\frac{c_{k}}{b_{k}} \\
P_{n}(x)=\frac{1}{2^{n}(n !)} \cdot \frac{\mathrm{d}^{n}}{\mathrm{~d} x^{n}}\left(x^{2}-1\right)^{n} \\
c_{1}=x-a \\
c_{2}=x=c_{3} \\
c_{4}=x+a
\end{array}\right.
$$

\subsection{Design of the Multi Solenoid Type Magnet}

Based on the actively shielding theory of SMES, a new structure magnet for 1 MJ HTS SMES is designed as Figure 5, where each solenoid has the same size. The direction of current in magnet units are illustrated as this Figure.

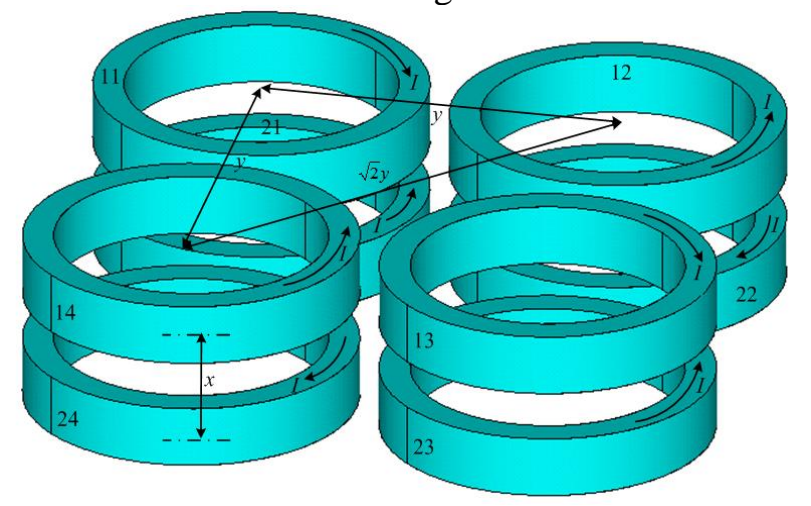

Figure 5. Model of Multi Solenoid Type Magnet

As shown in the figure, all of the solenoid units are numbered. The inductance of the multi solenoid type magnet $L_{\mathrm{m}}$ can be expressed as follow 


$$
L_{\mathrm{m}}=8 L+16 M_{1}-8 M_{2}-8 M_{11}-16 M_{12}+8 M_{13}
$$

where $L=$ self-inductance of the solenoid unit; $M_{1}=$ mutual inductance between NO. 11 and NO. 12 solenoid; $M_{2}=$ mutual inductance between NO. 11 and NO. 13 solenoid; $M_{11}$ $=$ mutual inductance between NO. 11 and NO. 21 solenoid; $M_{12}=$ mutual inductance between NO. 11 and NO. 22 solenoid; $M_{13}=$ mutual inductance between NO. 11 and NO. 23 solenoid. In addition, the other mutual inductance can also be expressed by $M_{1}, M_{2}, M_{11}$, $M_{12}$ or $M_{13}$.

\subsection{Optimization of SMES Magnet}

4.3.1. Optimization of single solenoid type magnet: The proposed objective functions, minimum volume of single solenoid type magnet, can be expressed as

$$
V=\pi d a r=\min
$$

equation (14) subjects to

$$
\left\{\begin{array}{l}
L_{\mathrm{s}}=2 \\
R_{1} \geq 0.1 \\
a<d \\
R_{1}=\frac{d-r}{2} \\
R_{2}=\frac{d+r}{2} \\
a=0.0044 \cdot \omega \cdot \frac{0.0054}{R_{2}-R_{1}}
\end{array}\right.
$$

4.3.2. Optimization of multi solenoid type magnet: The proposed objective functions, minimum volume of multi solenoid type magnet, can be expressed as

$$
V=8 \pi d a r=\min
$$

equation (16) subjects to

$$
\left\{\begin{array}{l}
L_{\mathrm{m}}=2 \\
R_{1} \geq 0.1 \\
a<d \\
\frac{\sqrt{2}}{2} y \geq \sqrt{2} \frac{d+r}{2}+0.02 \\
x \geq a+0.02 \\
R_{1}=\frac{d-r}{2} \\
R_{2}=\frac{d+r}{2} \\
a=0.0044 \cdot \omega \cdot \frac{0.0054}{R_{2}-R_{1}}
\end{array}\right.
$$


4.3.3. Optimal results: By using particle swarm optimization, the structural parameters of two kinds of magnets can be optimized respectively. The optimal results are shown as Table 1.

Table 1. Optimal Results of Two Kinds of Magnets

\begin{tabular}{lllllll}
\hline & $R_{1}(\mathrm{~m})$ & $R_{2}(\mathrm{~m})$ & $a(\mathrm{~m})$ & $x(\mathrm{~m})$ & $y(\mathrm{~m})$ & $V\left(\mathrm{~m}^{3}\right)$ \\
\hline single solenoid type magnet & 0.3874 & 0.4623 & 0.1584 & - & - & 0.0313 \\
multi solenoid type magnet & 0.1911 & 0.2741 & 0.0704 & 0.0989 & 0.5780 & 0.0683 \\
\hline
\end{tabular}

\section{Analysis of Magnetic Field}

According to the optimal results, the ANSYS modeling simulations of the two magnets are built respectively. The magnetic flux density vector images of two magnets are shown as Figure 6.
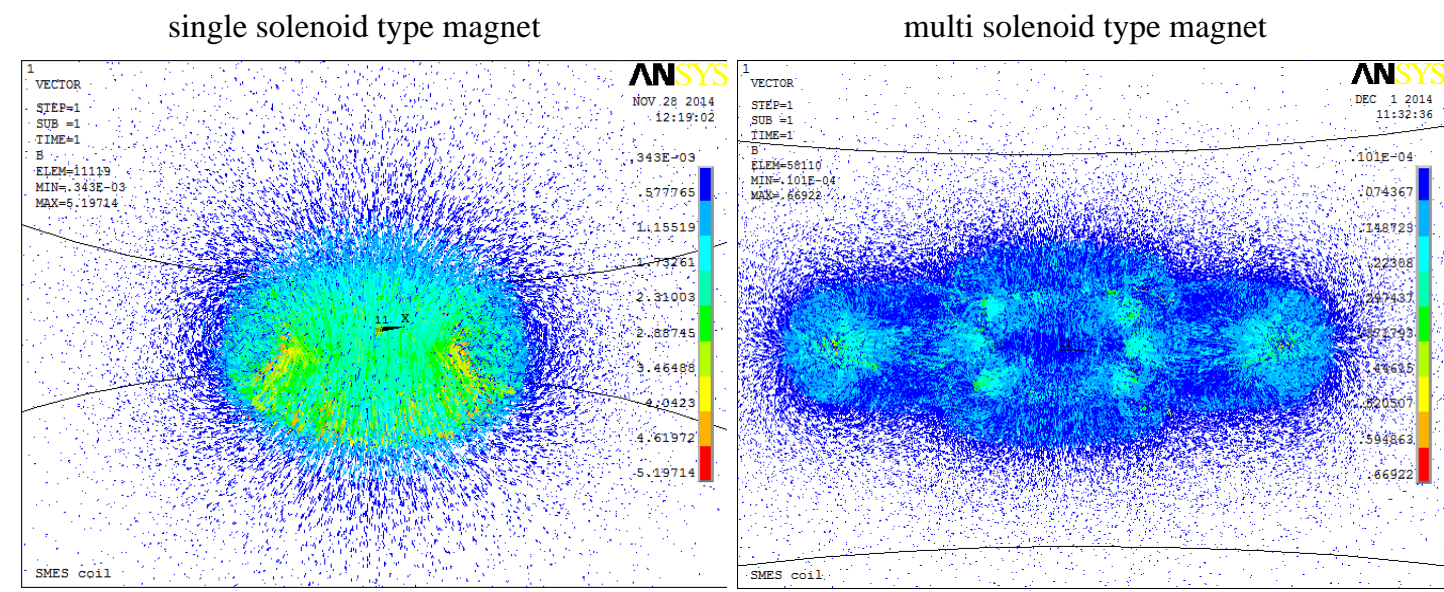

Figure 6. Magnetic Flux Density Vector Images of Magnets

In order to visually illustrate the reduction trend of magnetic field, we draw the section of magnetic flux density respectively in the middle of magnet. The images are shown as Figure 7. 


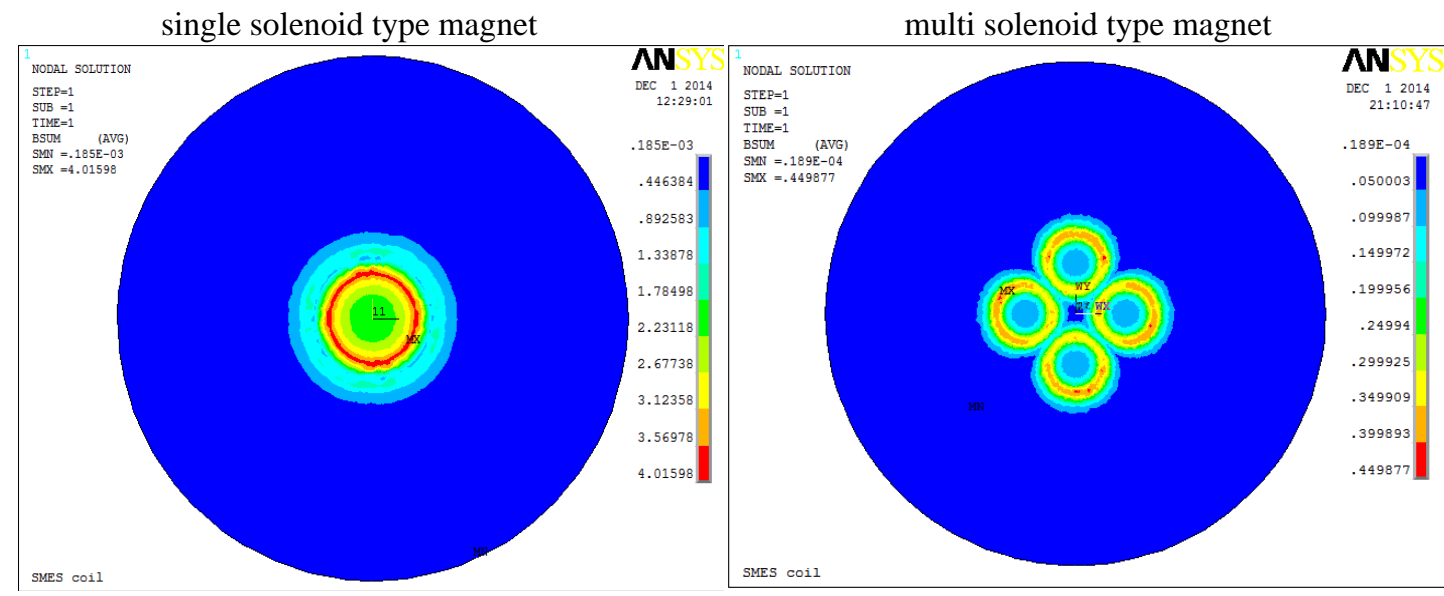

Figure 7. Section of Magnetic Flux Density

As shown in Figure 6-7, magnetic flux density around the multi solenoid type magnet decreases largely compared to that around the single solenoid type magnet. The leakage magnetic field of multi solenoid type magnet decrease rapidly. By extracting magnetic flux density around two magnets, the maximum magnetic flux density with a distance of 2 $\mathrm{m}$ is shown as Table 2. It's turns that the multi solenoid type magnet has weaker leakage magnetic shielding.

Table 2. Maximum Magnetic Flux Density with a Distance of $\mathbf{2} \mathbf{~ m}$

\begin{tabular}{ll}
\hline & magnetic flux density $(\mathrm{T})$ \\
\hline single solenoid type magnet & $0.962 \times 10^{-2}$ \\
multi solenoid type magnet & $0.711 \times 10^{-3}$ \\
\hline
\end{tabular}

\section{Conclusions}

In this paper, we propose a new structure magnet for 1 MJ SMES. Based on the actively shielding theory, the multi solenoid type magnet is designed. By using particle swarm optimization, the structural parameters of single solenoid type magnet and multi solenoid type magnet are optimized respectively. According to these parameters, the ANSYS modeling simulations of two magnets are built. The magnetic flux density from two magnets are contrasted. We can find that the maximum magnetic flux density with a distance of 2 $\mathrm{m}$ of the multi solenoid type magnet gets $92.61 \%$ reduction compared to that of the single solenoid type magnet. It's turns that the multi solenoid type magnet has effect on leakage magnetic shielding by consuming more HTS wires.

\section{References}

[1] U. Bhunia, S. Saha and A. Chakrabarti, "Pareto optimal design of sectored toroidal superconducting magnet for SMES", Physica C: Superconductivity, vol. 5,no. 5, (2014), pp. 6-13.

[2] G. Fresacher and G. Schonwetter, "Actively shielded transportable SMES-systems", IEEE Transactions on Magnetics, vol. 32,no. 4, (1996), pp. 2349-2352.

[3] Guru, B. S., and Hiziroglu, H. R., Editor, Electromagnetic field theory fundamentals, Cambridge University Press, Cambridge (2004)

[4] T. Hamajima, M. Shimada and S. Hanai, "SMES coil configurations with reduced stray field", IEEE Transactions on Applied Superconductivity, vol. 9,no. 2, (1999), pp. 346-349. 
[5] W. Hassenzahl, "Considerations against a force compensated coil", IEEE Transactions on Magnetics, vol. 25,no. 2, (1989), pp. 1854-1857.

[6] Калантаров, П. Л. and Пейтлиц, Л. А., Editor, The Inductance Calculation Handbook, China Electric Power Press, Beijing (1957)

[7] WS Kim, SY Kwak and JK Lee, "Design of HTS magnets for a $600 \mathrm{~kJ}$ SMES", IEEE Transactions on Applied Superconductivity, vol. 16,no. 2,(2006), pp. 620-623.

[8] S. Noguchi and H. Yamashita, "An optimization method for design of SMES coils using YBCO tape", IEEE Transactions on Applied Superconductivity, vol. 13,no. 2, (2003), pp. 1856-1859.

[9] G. Schonwetter, "SMES solenoids with reduced stray field", IEEE Transactions on Magnetics, vol. 30,no. 4,(1994), pp. 2636-2639.

[10] M. Song, "100kJ/50kW HTS SMES for Micro-Grid", IEEE Transactions on Applied Superconductivity, vol. 25,no.3, (2015), pp. 3785-3790.

\section{Authors}

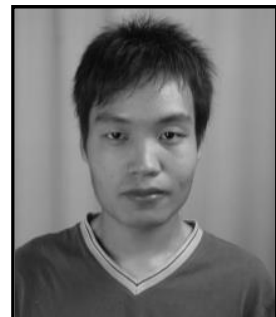

Wei Wei, He is currently pursuing his Master in Electrical Engineering in Lanzhou jiaotong University, China. His research interests include electromagnetic compatibility and SMES. 